\title{
To Be Continued
}

The Genidentity of Physical and Biological Processes

Alexandre Guay and Thomas Pradeu

In Guay, A. \& Pradeu, T. (eds.) Individuals Across the Sciences. New York:

Oxford University Press, 2015, ISBN 978-0-19-938251-4, p. 317-347.

\subsection{Introduction}

In 1922, Kurt Lewin (a leading German-American psychologist, 1890-1947) proposed the concept of "genidentity" to better understand identity through time. Lewin's aim was to offer a conception of identity that would be relevant across different sciences, especially physics and biology (Lewin 1922). Later, philosopher Hans Reichenbach (1891-1953) distinguished different conceptions of genidentity, and applied them to physical cases (Reichenbach [1956] 1971). However, many philosophers and scientists have considered that Reichenbach's view of genidentity was imprecise and, consequently, failed to shed light on the identity of physical objects. In particular, Reichenbach's account does not seem to apply to particles in nonrelativistic quantum mechanics, a domain in which trajectories and causation are problematic (e.g., French and Krause 2006, 48-49). Recently, a few philosophers have resorted to the concept of genidentity to reflect on mereology (e.g., Smith and Mulligan 1982), or to understand biological entities (e.g., Boniolo and Carrara 2004), but their views have not aroused much discussion in the philosophical community. In this chapter, examining several specific examples taken from current classical physics and biology, we defend the genidentity view and show that it would be fruitful to adopt it in at least some areas of those sciences.

What does the concept of genidentity say? In a nutshell, it says that the identity through time of an entity $\mathrm{X}$ is given by the continuous connection of states through which $\mathrm{X}$ goes. For example, a "chair" is to be understood in a purely historical way, as a connection of spatiotemporal states from its making to its destruction. In this view, the individual X is never presupposed or given initially, because the starting point is the decision to follow a specific and appropriate process $\mathrm{P}$, and the individual $\mathrm{X}$ supervenes on this process. For example (as detailed below), one can decide to follow the conservation of an internal physiological organization through time, and the effectuation of this process gives us our individual entity (in this case, an individual organism). In other words, for the genidentity view, what we single out as an "individual" is always the byproduct of the activity that is being followed, not its prior foundation (not a presumed "thing" that would give its unity to this activity). (Of course not every sequence of events is associated with an individual, as explained below.) A bacterium must be seen as a connection of spatiotemporal states: these states are "genidentical." Now, if a bacterium divides into two daughter bacteria, most biologists will say that this division marks a new start, which means that states of the mother bacterium and states of the daughter 
bacteria cannot be considered as "genidentical." The statement that "a new start" occurs, which is the key statement of the genidentity view, naturally depends on a criterion of continuity adopted by the observer. We show below how experimental sciences can help us define these criteria in specific contexts.

The genidentity view can be better understood by its opposition to other conceptions of temporal identity. First, the genidentity view is antisubstantialist since it says that the identity of X through time does not presuppose that "something" of X remains. (It is also-perhaps even more explicitly-antiessentialist, at least if essentialism is understood as the claim that X is the same if there exists a permanent "core" or "substrate" of X through time.) Indeed, in the genidentity view, the question "What is X, fundamentally?" is replaced by the question "How should I follow X through time?" Second, the genidentity view is also in opposition to the idea of identity-resemblance, according to which $\mathrm{X}$ remains through time if it sufficiently resembles itself. In other words, three general conceptions of identity through time are schematically distinguished here:

1. Substantialism: the identity of $\mathrm{X}$ is rooted in the idea that something of $\mathrm{X}$ remains through time (and essentialism is therefore one important form of substantialism, though not the only one: as will be explained below, Leibniz and Wiggins, for instance, are substantialists but not essentialists).

2. Identity-resemblance: the identity of $\mathrm{X}$ is rooted in the idea that $\mathrm{X}$ looks sufficiently like itself through time.

3. Genidentity: the identity of $\mathrm{X}$ is rooted in the idea that $\mathrm{X}$ is characterized by sufficiently continuous states through time.

As has often been recognized (e.g., Boniolo and Carrara 2004, 456 n. 1), the genidentity view draws on the conception of identity defended by Locke ([1694] 1975). Exploring the problem of identity through time of a given entity (be it a tree, an animal, or a human), Locke considers that the most satisfying criterion for diachronic identity (the "principle of individuation") is continuity of states (1975, II, 27, §3, 330). Applied to the problem of the identity through time of a given man, this conception leads Locke to assert:

This also shows wherein the Identity of the same Man consists; viz. in nothing but a participation of the same continued Life, by constantly fleeting Particles of Matter, in succession vitally united to the same organized Body. (Locke 1975, §6, 331)

Our intention is to build further on Locke's suggestion, in order to offer a precise definition of the notion of genidentity, applicable to physics and biology, and to lay the foundations of an ontology centered on processes and change rather than substances, invariance, and laws. Famously, Leibniz (in particular in his New Essays, written as a systematic response to Locke), disagrees with Locke on identity viewed as continuity:

By itself continuity no more constitutes substance than does multitude or number. ... Something is necessary to be numbered, repeated and continued. (Leibniz [1765] 1916, Gerhardt II, 169)

In contemporary metaphysical debates about identity, David Wiggins $(2001,57)$ 
explicitly endorses Leibniz's view, and rejects the idea that identity could be defined as bare continuity (see also Wiggins 1968). The dispute between Locke and Leibniz, therefore, is far from being extinguished. Here we defend a view close to Locke's, in some specific cases taken from physics and biology (a similar defense, applied specifically to immunology, can be found in Pradeu and Carosella 2006).

Our focus in this chapter is on diachronic, not synchronic, identity: we are not asking what makes the identity of a physical or biological object at time $t$-for instance how it can be distinguished from other things, what its physical boundaries are, and so on. Instead, we are asking what makes X the "same" at two different moments in time. This will naturally involve questions about distinguishability, boundaries, or individuality, but always in a temporal, historical context. In other words, to use the language of relativity, we will not discuss why a particular event can be associated to a particular individual, but how different events can be related and seen as characterizing the same individual. ${ }^{1}$

Though the intention of its instigators was to make a useful contribution to experimental sciences, applications of the concept of genidentity to real science have been scarce and, usually, unsatisfying. In this chapter, we try to take up Lewin's objective by demonstrating that a better-defined concept of genidentity sheds an important light on identity through time both in physics and in biology.

We are aware that a direct comparison between physics and biology regarding the way they conceptualize identity may seem surprising. Physics and biology both raise questions about individuality and identity through time, but they do so in a markedly different way. These differences include the four following aspects:

1. In biology, parts-whole questions seem crucial, probably because most, if not all, biological entities appear as constituted of smaller biological entities (as, for example, when one asks to what extent the cells constituting a multicellular organism are themselves "individuals"), and most biological entities appear to be constituents of larger biological entities, while part-whole questions play a less important role in physics. ${ }^{2}$

2. In physics, distinguishing one particle among many identical particles is a key issue, while in biology even individuals that are said to be "identical" express, most of the time, some significant differences and, at the very least, can usually be spatially distinguished.

3. In physics, the principle of indiscernibles is critical in discussions about synchronic identity, but not in biology. Biologists often say that two living things are "identical" even when they do not share all their properties (in particular their position in space), as with clonal organisms.

4. In physics, discussions over structuralism are extremely important, as one crucial aim is to determine what remains invariant under transformations; in biology, structuralism plays a lesser role, if any (for exceptions to this trend, see French 2011, French and Ladyman, this volume). 
Despite these important differences, we intend to show in this chapter the utility of a comparison between physics and biology in their understanding of identity through time, and the fruitfulness of the concept of genidentity in both domains. From our point of view, the gain is more significant in biology than in physics, but some important cases taken from physics can nonetheless benefit from the adoption of a genidentity view. In other words, we suggest that the genidentity view is useful both in biology and in physics, and, perhaps even more importantly, that it can be pivotal to fostering a dialogue between these two major scientific fields. The outline of the chapter is as follows. In section 16.2, we examine different concepts of genidentity and the difficulties they raise. In sections 16.3 and 16.4, we explore several examples, respectively in physics and in biology, that demonstrate the utility of adopting the genidentity view. In section 16.5, we lay the foundations of an ontology of processes and change, in contrast to an ontology of substances, invariance, and laws.

\subsection{Approaches to Genidentity}

\subsubsection{Lewin's Conception of Genidentity}

The term "genidentity" is typically regarded as coined by Lewin in 1920 (and later analyzed in Lewin 1922), though he was not the first to use it in a published work. For Lewin, the concept of genidentity is expressed in mereological terms. A temporally extended entity has to be understood as a multiplicity of entities, all parts of the genuine individual, and the relation between these entities is genidentity. ${ }^{3}$ Since the kind of mereology involved in each scientific discipline is not exactly the same, the appropriate genidentity relation is therefore context dependent.

Lewin defines simple and complete genidentity. Two collections of temporal parts (sets of entities at two distinct times) are simply genidentical if they are genidentical but one of the collections (let us say the earlier one) could also be said to be simply genidentical to another collection at the second time. The two collections are said to be completely genidentical if that is not the case. This distinction will not play any role in this chapter but is important in Lewin's conception because simple and complete genidentity definitions are related. As shown by Smith and Mulligan (1982), in the context of physics, Lewin's definition of simple genidentity on the one hand and complete genidentity on the other can be formalized as shown in figure 16.1 (if we represent complete genidentity by $\mathrm{p} \equiv$, simple genidentity by $\mathrm{p}=$, is a proper or improper part of by $\leq$ and, is discrete from as /).

$$
\begin{array}{r}
a^{p} \equiv b:=\neg \exists x\left(x / a \& x^{p}=b\right) \& \neg \exists x\left(x / b \& x^{p}=a\right) \\
a^{p} \equiv b \rightarrow \forall x\left(x / b \rightarrow a^{p} \neq x\right) \& \forall x\left(x / a \rightarrow b^{p} \neq x\right) \\
a^{p}=b \rightarrow \exists a^{\prime} \exists b^{\prime}\left(a^{\prime} \leqslant a \& b^{\prime} \leqslant b \& a^{p} \equiv b^{\prime}\right)
\end{array}
$$

Figure 16.1 Formalization of simple and complete genidentity according to Smith and Mulligan (1982)

We may illustrate this definition with an example. Let us imagine that we have initially a 
piece of iron and a bucket of strong acid. The piece of iron falls in the bucket and dissolves, emitting heat. According to the above definition (iron + acid) $p \equiv$ (acid solution + heat) and (iron + acid) $\mathrm{p}=$ (heat). The mereological definitions (simple and complete genidentity) imply that after the action of the acid on the iron piece, the iron is still in the solution in a certain form. And before this action the heat was in a certain form present. All this is of course true: the iron is still present as atoms in the solution and the heat was present in the initial state as chemical binding energy. But obviously what is meant by "to be present" is very different in each case. This is an important disadvantage of any mereological definition of genidentity. In this approach, if two states are genidentical, they must be conceived as different parts of the same temporally extended entity. This assertion does not shed any light on the relation that makes these two states of the same thing. Moreover, we could argue that once we have individuated a temporally extended entity in order to identify its temporal parts, we do not need to give a precise definition of this relation since we already possess a diachronic identity criterion. In conclusion, Lewin's definition of genidentity presupposes that we already have a method to individuate individuals through time, which is highly problematic. In Lewin's account, genidentity is a primitive notion. Overall, therefore, this approach does not seem appropriate to develop an operative concept of genidentity that could be used in physics and biology.

\subsubsection{Reichenbach's Conception of Genidentity}

Reichenbach ([1956] 1971) offered his own conception of genidentity. It is this conception that will be applied here to biology and physics. (We will focus on the later work of Reichenbach on genidentity; on his earlier conception, see Padovani 2013.) Reichenbach distinguishes genidentity, which is applied to physical entities, from logical identity:

The physical identity of a thing, also called genidentity, must be distinguished from logical identity. An event is logically identical to itself; but when we say that different events are states of the same thing, we employ a relation of genidentity holding between these events. A physical thing is thus a series of events; any two events belonging to this series are genidentical. (Reichenbach [1956] 1971, 38)

In this quote, Reichenbach points exactly to the main difficulty inherent to the notion of genidentity. As Quine $(1966,145)$ later argued, the diachronic identity of a thing cannot be captured by logical identity alone, since such identity is indexed by spacetime locations; therefore, something else must be provided. ${ }^{4}$ Reichenbach believes that "speaking of things and speaking of events represent merely different modes of speech" ([1956] 1971, 224), and he proposes to define the diachronic identity of a thing as a relation among events. At a minimum, two genidentical events should be related by a "worldline" that lies in the light cone of the earliest event (in other words, two events cannot be genidentical if they can be related only by a signal going faster than the speed of light). Being related by a worldline is necessary for genidentity, but it is not sufficient, since there is a certain degree of arbitrariness in how we define worldlines for successive events (it is especially true in the case of fields, for which the direction of the striation is arbitrary). This arbitrariness is one of the reasons that convinced Einstein that the concept of substance was inadequate for modern physics (Reichenbach 1958, 270-271). 
In addition to being related by a worldline, then, two genidentical events must be related by a causal relation. ${ }^{5}$ But not any causal relation will do the trick. Only causal chains that could be said to relate events characterizing states of the same thing should be considered. Since there is more than one way to talk about the same thing through time, Reichenbach argues that there will be more than one way to define a genuine genidentical relation. That genidentity could be defined in multiple ways and, therefore, that the notion of an individual is relative to a certain theoretical point of view should not be surprising. In Reichenbach's (and, even more, in our) approach to the relation between metaphysics and science, metaphysical concepts, such as, for example, the concept of an individual, help us organize the scientific discourse, but they are certainly not a foundation to it. Except in cases where "individual" is a theoretical term defined explicitly in a theory, the concept of an individual could be abstracted or projected on the scientific discourse. It does not bind it, nor are we forced to include it in our ontology. However, ontological commitment can act as a constraint. Indeed, it could be argued that, in Reichenbach's work at least, the concepts of genidentity and causality are closely connected, to the point that the latter cannot be defined without the former. When Reichenbach discusses the constraints on a causal chain signal in special relativity, he conveys the idea that something is propagating through space and time. In consequence, a principle, namely genidentity, is required to individuate this something through space-time, in order to discuss the causal relation involved.

The closest way to define a genidentity relation that could reflect how we talk about ideal macroscopic objects, like billiard balls (if these objects were unaltered by any interaction), is what Reichenbach called material genidentity. The aim of this definition is to get to the diachronic identity of beings evolving temporally. This relation can be defined by three characteristics: (1) continuity of change, (2) spatial exclusion, and (3) distinguishability of states that differ only in the permutation of two objects ([1956] 1971, 225). The first characteristic expresses the fact that you must be able to follow the evolution of the individual through space and time. Continuity of change is the easiest way to achieve this goal, but this condition could be relaxed in certain cases. The second characteristic specifies that different individuals cannot occupy the same position simultaneously. This guarantees that an interaction between individuals will not make the identity ambiguous. The last characteristic is less intuitive but is justified, we think, by the need to avoid the identity ambiguity when the determination of which events are simultaneous could differ depending on the chosen reference frame. Of course, if no two individuals share all the same properties, they cannot be wrongly identified. This is not in general the case in physics. ${ }^{6}$ These characteristics are necessary but not sufficient to guarantee individuality, since, as Reichenbach admits, there are obvious counterexamples ([1956] 1971, 225).

In practice, it would be difficult to find any useful application of the notion of material genidentity in current physics. Nevertheless, it can work as an idealized (paradigmatic) case, illustrating the necessary conditions for a more complex and more useful conception of genidentity. In this second conception, we are interested in the evolution through space and time of a unique individual potentially interacting with other individuals at a distance. To capture such a process, Reichenbach proposes the concept of functional genidentity, which refers to the retention of the same "function" through 
time, without retention of matter. For instance, if we claim that an organism is still the "same" after absorbing some nutriments, we are considering the identity through time of an individual that interacts with other individual entities, and, this is not something that material genidentity can explain (because material genidentity presupposes spatial exclusion) (Reichenbach [1956] 1971, 227); we need to take into account the "functional" identity of this organism, and not its "material" identity. Reichenbach remains imprecise about "functional genidentity," but he does give a nice example: transversal water waves. In a transversal wave, water moves only vertically. The continuity of change does not involve transportation of matter. However, there is a continuous momentum and energy propagation perpendicular to this vertical move. Spatial exclusion is violated, since waves can superpose and there could be permutation symmetry since a wave can be identical to another if they share the same dynamical properties. Nevertheless, in many circumstances, you can track a wave in space-time by defining a function of the wave parameters that evolves continuously.

Which function should be used to track the "real" individuals is not an appropriate question in this context. Of course, as a minimal requirement, the function should denote a causal process. But among all possible causal processes, which one should be understood as relating states of the same thing is a question without a general answer. Someone could claim that the wave equation describes the collective behavior of water molecules, the legitimate individuals or, even further, that the wave is denoting the change in space-time points' properties, which would be the "real" individuals. Since the connection between a scientific theory and its ontology is not straightforward, all these positions can be argued (Braillard et al. 2011). In section 16.3, we return to the question of whether the existence of a particular function is enough to claim that a wave is an individual.

Overall, Reichenbach has made an important contribution to the definition of genidentity by making several key distinctions:

1. Genidentity is about physical identity, which implies more than just logical identity.

2. Genidentity corresponds not to any physical identity (defined as a worldline of continuous states), but to causal physical identity (defined as a worldline of causally connected continuous states).

3. Genidentity can be divided into material genidentity (defined as retention of matter, distinguishability, and spatial exclusion with regard to other entities) and functional genidentity (defined as retention of the same "function" through time, without retention of matter).

After Reichenbach, very few scientists and philosophers have used the notion of genidentity. (There are some exceptions, including Boniolo and Carrara 2004, but because of space limitation we will not discuss them here.)

\subsection{Genidentity in Physics}

In this section, we show how the notion of genidentity can shed light on the problem of individuation in classical physics. Importantly, we will not adopt here a normative approach to the notion of an individual. In particular, we will not claim that only something like material genidentity (defined as retention of matter, distinguishability, 
and spatial exclusion with regard to other entities) captures the real concept of an individual (see Table 16,1), although it is undeniable that this relation is very close to the concept we use in ordinary life when talking about the identity of material things. Instead, we will start with material genidentity, and then relax its requirements in order to generate a more applicable concept of genidentity, namely a functional one. Armed with this functional genidentity relation, we will, in section 16.4, apply it to a muchdebated problem, the problem of biological individuality.

Table 16.1 Necessary Conditions for Material Genidentity

\begin{tabular}{|c|c|c|}
\hline \multicolumn{3}{|c|}{ Material Genidentity } \\
\hline 1) Continuity of change & 2) Spatial exclusion & 3) Distinguishability \\
\hline
\end{tabular}

This move from material genidentity toward functional genidentity will be structured by three paradigmatic cases of growing complexity, each step building on the previous one: (1) the free individual (the form of functional genidentity that is closest to material genidentity), (2) the changing individual without interaction, and finally (3) the fully interacting individual (see table 16.2). Once the three types are described, we can combine them to represent complex situations. All these cases will be illustrated in wave theory. The choice of wave theory is not insignificant. Contrary to particle physics, undulatory mechanics has traditionally been hostile to the concept of an individual. So offering a definition of an individual in this context would demonstrate the fruitfulness of our approach. Though philosophers of physics have very rarely paid attention to this aspect of wave theory and to the case of solitons in particular, our conviction is that this is a pivotal domain to understand the notion of genidentity and, more generally, to reflect on identity in classical physics. It is worth mentioning that, since the standard procedure to get to a quantum theory is to start from a classical theory, even if the ontology of a particular quantum theory could be different from its associated classical theory, it is never totally independent from it.

Table 16.2 Paradigmatic Cases of Functional Individuals

\begin{tabular}{|ccc|}
\hline Free functional individuals & $\begin{array}{c}\text { Relaxed characteristics } 2 \text { and } \\
3 \text { of material individuals }\end{array}$ & Ex.: free solitons \\
\hline $\begin{array}{c}\text { Changing functional } \\
\text { individuals }\end{array}$ & $\begin{array}{c}\text { Constant or continuously } \\
\text { changing rate of the function } \\
\text { defining free individuals }\end{array}$ & Ex.: dissipating waves \\
\hline $\begin{array}{c}\text { Interacting functional } \\
\text { individuals }\end{array}$ & $\begin{array}{c}\text { Changing/free individuals }+ \\
\text { interaction events (with or } \\
\text { without loss of identity) }\end{array}$ & Ex.: interacting solitons \\
\hline
\end{tabular}

Let us start with the first case, the free individual. A perfect billiard ball moving in a straight line in vacuum is a model of a free individual for which the states are materially genidentical. If we relax the second characteristic (spatial exclusion) of material genidentity, a soliton, that is, a kind of self-reinforcing solitary wave, becomes as well a model close to material genidentity (Drazin and Johnson 1989). A soliton is a localized traveling wave in nonlinear systems. It does not obey the superposition principle and does not dissipate. In the case of a soliton, no matter is globally transported. As Reichenbach had emphasized for wave theory, the diachronic identity of a soliton can 
only be represented by functional genidentity. For example, the solution of the nonlinear partial differential equations could serve as the identity function since the shape of the wave is unchanging and the movement is continuous. Other functions of the topology or conservation principles could also be used. Our claim here is that a soliton is functionally as close as one could get to a substantial individual for which we would have a material genidentity relation enabling us to follow its states. Trivially, a solitary soliton is absolutely discernible and it can be continuously followed. For us, it is the paradigmatic case of a functional individual. As to the question "Is this particular soliton solution representing a physical individual or not?," it does not have a general answer. All depends on how this particular piece of mathematics is used in the physical model. It is the physical model that defines what kinds of functions are ontologically significant for diachronic identity. It is worth mentioning that solitons are considered as particles or more precisely quasi-particles in many quantum field models. These particles are different from the so-called elementary particles since they do not arise from the quantization of the wavelike excitations of the fields and possess a topological structure (Mantan and Sutcliffe 2004).

Let us now return to the case of the billiard ball in a vacuum, and relax the third characteristic (distinguishability under permutation) in order to allow other free identical billiard balls moving around without interacting one with the other. The equivalent model would be many identically shaped solitons that travel without meeting or interacting one with the other. In this case, our entities are not absolutely discernible anymore but are at best weakly discernible (for definitions, see Saunders's and Ladyman's chapters in this volume). Should we in this context renounce calling them "individuals"? We do not think so. Only if a change of reference frames implies an identity ambiguity among them should we renounce talk about "individuals." In many cases, the loss of absolute discernibility does not preclude the definition of a robust genidentity relation, since each soliton could be followed precisely in space-time.

Let us now discuss the second case: the continuously changing individual. Here we have in mind cases where an individual is apparently changing during its movement in space-time, for example, a billiard ball continuously changing color, or, in undulatory theory, a continuously changing solitary wave. The first case, the free individual, gives a baseline with which to understand this new case. We have a function that allows us to follow the individual through its continuous evolution, typically with one or many conserved quantities. Now we allow this function to fluctuate. We measure its derivative to quantify this change. Under which rate of change we should still consider the related events as characterizing the same individual is a contextual question. Note that we do not ask how much color change, or topology change, is enough to talk of a new individual, but how intense, or discontinuous, the rate of change must be to assert we face a new individual or no individual at all. The question of color or topology change presumes that the identity of the billiard ball is essentially defined by the possession of some properties. The second and third questions, in contrast, are in the spirit of the genidentity conception of the individual. No properties or structure define the identity of the billiard ball or of the wave beyond the causal process followed by the genidentity relation. Please take note that in this case, the source of the change (color, shape...) is not on the same ontological level as the studied individual. The source of change is considered diffused in the environment. There is no clear vertex with the worldline of 
another individual. This is the main difference with the next and final case.

Our final case is the fully interacting individual. In the genidentity approach, this case is the really challenging one, since no change of essential properties can inform us when an interaction destroys or creates an individual. Only a careful study of the nature of the interaction itself can provide elements for discussing the identity of the individuals involved. To illustrate this point, let us look at the interaction between a green and a red billiard ball. In the initial state, we have two balls coming toward each other. For the sake of the discussion, let us suppose that we do not have access to the details of this particular interaction. In the last stage, we see a green ball and a red ball apparently identical to the incident balls going away from each other with a certain angle compared to the initial trajectories. What can we say about the conserved identity of the individuals involved? If the interaction is a simple repulsion, it seems probable that the final green ball is genidentical to the initial green ball, and the same would be true for the red one. However, if the repulsion is accompanied by an exchange of color, our answer would be different. The nature of the interaction is what guides us in our assessment of the billiard balls' identity. Of course, if the genidentity of billiard balls is of the material kind, then each individual is uniquely associated to a nonintersecting spacetime trajectory; therefore we simply have to follow their trajectories during the interaction to be able to know which is which. But in a case of functional genidentity, where spatial exclusion is not a given, the study of the nature of the interaction cannot be put aside.

This last case is precisely the subject of the study of soliton interactions. Contrary to linear waves, which do not interact when they are superposed, the nonlinear nature of solitons implies complex interactions. Only a systematic study of these interactions allows us to say something about the genidentity of interacting solitons. For example, let us take the class of one-dimension line solitons involved in a nonresonant elastic interaction. In these situations, the number of solitons is conserved, except during the interaction phase. All soliton properties are completely restored after interaction. For example, the direction of propagation and the linear momentum seem unchanged. Important amplitude change can occur during the interaction. For example, in many cases the total amplitude of the superposed solitons is less than the sum of each individual amplitude, but each amplitude is restored after the interaction (see figure 16.2). The interaction often generates a phase shift, a change of position compared to what would be the position of the solitons if the interaction did not occur (for more details, see Soomere 2011). Depending on the particular type of functional genidentity that is discussed, these characteristics of the interaction will or will not allow us to assert that the solitons emerging from the interaction are the same as the ones entering into it. More precisely, the question that we need to ask is in what way the interaction perturbs the genidentity established in the second paradigmatic case. In these examples, even if the interaction is complex, it seems that the worldline of a particular soliton is almost not perturbed except for a phase shift. It seems reasonable to argue that, for most functional genidentity relations, the initial individuals are the ones appearing in the final state. However, this is not the only possible case. In the context of resonant interaction, the fusion of two or more solitons in a new soliton is possible. The time reverse process, fission, is also possible. Obviously, in this case, the initial solitons do not survive. Some cases are more ambiguous and therefore difficult to decide. 

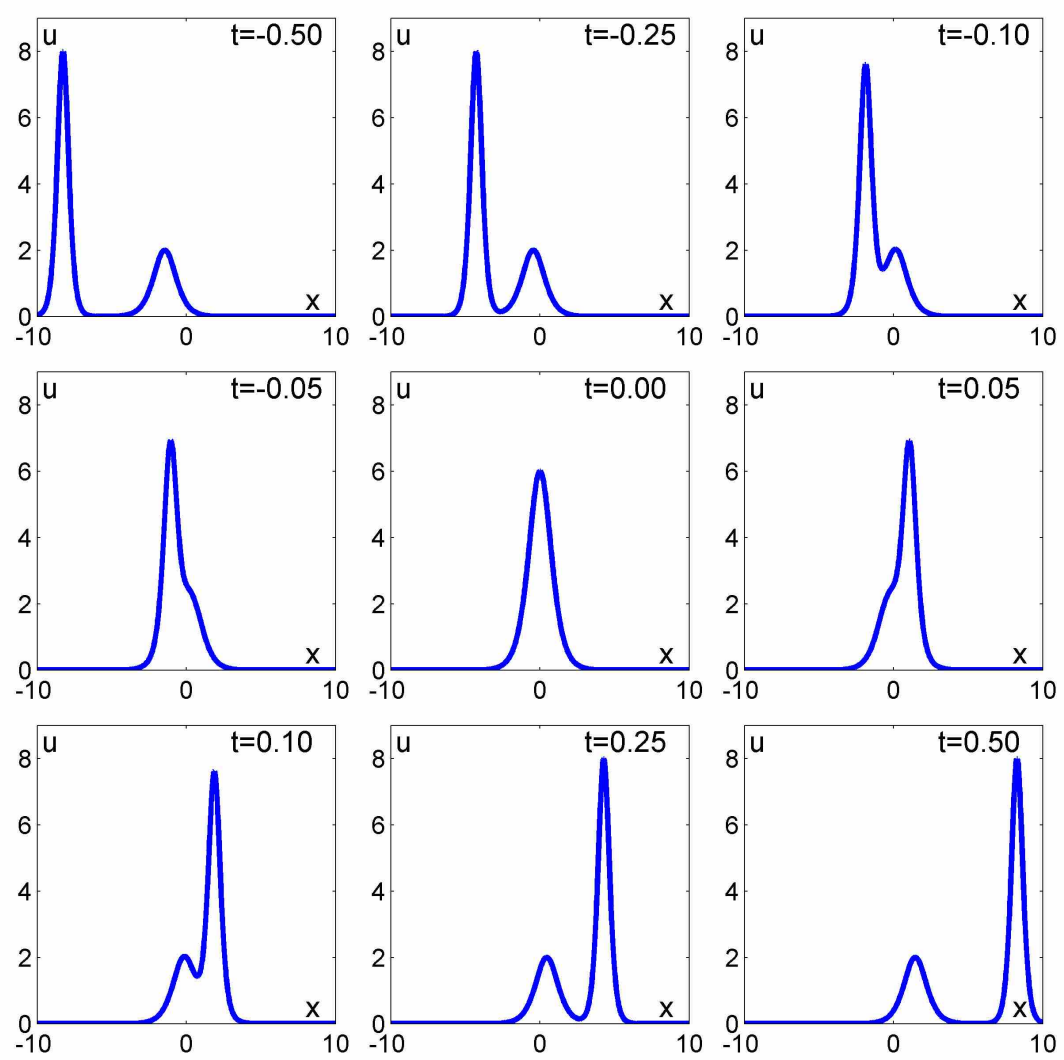

Figure 16.2 Temporal evolution of Korteweg-de Vries solitons described by the two-soliton solution $\mathrm{u}(x$, $t)=12 *(3+4 \cosh (2 x-8 t)+\cosh (4 x-64 t)) /\left([3 \cosh (x-28 t)+\cosh (3 x-36 t)]^{2}\right)$ (Soomere 2011, 1583). Note the nonadditivity of amplitudes during interaction With kind permission from Springer Science and Business Media.

This point can be illustrated by an example, that of head-on collisions of two baby skyrmions (BS). Baby skyrmions are topological solitons, in a $(2+1)$-dimensional field theory, which are closely related to the Skyrme model. In a nutshell, a skyrmion is a soliton solution to the Skyrme model; this field model is used, among other things, to represent states of baryons and excited baryons: see (Skyrme 1962). If the parameters are chosen to allow a head-on collision, at the apex of the interaction the two initial baby skyrmions merge to form a ring-like structure. Just after this, two new baby skyrmions emerge, for almost all initial parameters, at $90^{\circ}$ from the original incident trajectories. During all the interaction process, radiation is emitted (for more details, see Piette, Schroers, and Zakrewski 1995). Even if the initial BSs are not identical and the final two BSs are also not identical in a similar way, the symmetry of the interaction seems to limit our capacity to argue either way about the survival of the initial BSs. The complexity of the interaction is a strong limitation on the kind of functional genidentity relation that we could reasonably use. This contextuality of identity may seem problematic. If we could accept it for quasi-particles and biological beings, surely the identity criterion of fundamental particles should not be contextually defined? This worry presumes that there is such a thing as a fundamental theory describing fundamental entities. If, as it seems currently probable, we do not have a fundamental theory but only effective ones (see note 2), contextuality is unavoidable. This conclusion does not necessarily lead to arbitrary identities or pseudoidentities. It suggests that identity is relative to a theoretical context, for example, to a certain range of physical parameters shared by 
most theories, like energy.

Thus, in this section we argued for three points:

1. It is possible in a field theory to define a strong notion of genidentity, at least when worldlines can reasonably be defined. Conservation of matter is not necessary.

2. It is possible to define relaxed genidentity relations that could accommodate changing individuals.

3. Conservation of genidentity in interacting situations depends on the details of the interaction. More precisely, conservation of genidentity depends on how this interaction modifies the rate of change of the individuating function.

Overall, this section has shown that the notion of genidentity suggested here can be applied to some interesting physical cases, and that the confrontation with such cases can in turn help us to refine our notion of genidentity, and to better determine its scope. In the next section, we examine whether the notion of genidentity can be applied to biological cases, and we use what has been shown here about physical cases to shed a new light on the understanding of the identity of biological entities through time.

\subsection{Genidentity in Biology}

The previous section has presented several applications of the concept of genidentity to physical cases. We would now like to show that the concept of genidentity can also be applied to some biological cases or, more exactly, that it is the concept best suited to understand identity through time in biology.

Though questions pertaining to synchronic identity are important in biology, those pertaining to diachronic identity have long been recognized as central (Sober 2000, 154), in particular, at different temporal scales, in developmental biology and evolutionary biology. How do organisms and species remain the "same" through time, even though they change constantly? How do they "start" and "end" their lives? How can we know that we are talking about the "same" organism or the "same" species at two different moments in time, in particular if massive changes occur between these two moments?

Certainly more than any other philosopher of biology, David Hull (1935-2010) has recognized the crucial importance of the question of diachronic identity in biology. Even more strikingly, Hull explicitly defended the concept of genidentity in several texts. For Hull, a fundamental characteristic of living things is that they can undergo massive changes and nonetheless maintain their "identity." It is precisely this idea, Hull claims, that only the concept of genidentity can properly capture:

Three traditional criteria for individuality in material bodies are retention of substance, retention of structure, and continuous existence through time (genidentity). If organisms are to count as individuals, then the first two criteria are much too restrictive. In point of fact, many organisms totally exchange their substance several times over while they retain their individuality. Others undergo massive metamorphosis as well, changing their structure markedly. If organisms are paradigm individuals, then retention of 
neither substance nor structure is either necessary or sufficient for continued identity in material bodies. The idea that comes closest to capturing individuality in organisms and possibly individuals as such is genidentity. As its name implies, this criterion allows for change just as long as it is sufficiently continuous. The overall organization of any entity can change but it cannot be disrupted too abruptly. (Hull 1992) ${ }^{7}$

Elsewhere Hull expresses a similar defense of the concept of genidentity (Hull 1986). ${ }^{8}$ One might be surprised by the fact that Hull, arguably the most influential of all philosophers of biology in the twentieth century, explicitly used this notion to express one of his most famous theses (about the identity of living things) without having aroused much enthusiasm. Indeed, as we said in the beginning of this chapter, the notion of genidentity is rarely used by philosophers, philosophers of biology included (as an illustration, "genidentity" has only two occurrences, from 1986 to today, in Biology and Philosophy, arguably the leading journal in philosophy of biology), and even Hull did not change that situation. Is it because, here again, the notion of genidentity would be too imprecise? We believe, on the contrary, that Hull offers a rather precise and inspiring conception of genidentity, and that the reason that this conception has not been more successful lies elsewhere, namely, in its unusual metaphysical implications. Our strategy here, therefore, will be to reconstruct Hull's conception of genidentity, and then to extend it through an examination of several biological cases and a comparison with the uses of genidentity in physics.

In our view, what is probably Hull's most famous paper (Hull 1978) offers one of the best possible argumentations in favor of biological genidentity. This article is often seen as a defense of the theses that species should be seen evolutionarily as individuals rather than classes and that, consequently, there is no "human nature," no "essence" of humanity, if humans are understood as members of the species Homo sapiens (on Hull's and Ghiselin's "individuality thesis," see Haber, this volume). These theses are undeniably important, but we believe that the most crucial component of this paper is the series of diagrams drawn by Hull, and the conception of identity upon which they depend, that is, genidentity. Hull seeks a criterion of identity through time for living things. His thesis is that any organism or any species is a space-time portion, a "branch" on the tree of life, with a beginning and an end, with, between the two, a continuous line of different states. Hull argues that, because living things can undergo massive and unpredictable change, sameness and self-resemblance are inappropriate criteria for biological identity (Hull 1978, 345). He recalls that certain stages in some organisms are so different that biologists had placed them in different species, genera, families, and classes, before they could realize that those stages were in fact the transformations of one and the same organism. For living things, being the same cannot mean looking like oneself, and therefore the only possible criterion for identity in biology is continuity of change ("Phenotypic similarity, says Hull, is irrelevant in the individuation of organisms.")

Hull's diagrams offer a description of structural patterns of change in the living world, applicable to both organisms and species, because organisms and species belong to the same ontological category insofar as they both are spatiotemporally localized living things. A first set of structural changes concerns change of a living entity, or its splitting 
into two living entities (see figure 16.3 and table 16.3). What is Hull's criterion to postulate the potential emergence of a new entity? His criterion is the degree of disruption of internal organization. Of course, this criterion is not always easy to apply, and by

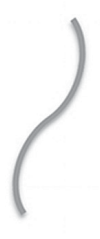

$1 \mathrm{a}$

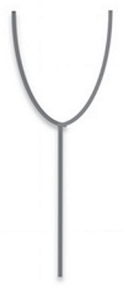

$1 \mathrm{~b}$

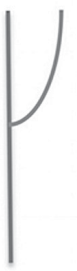

$1 c$

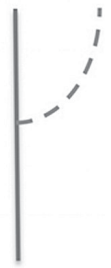

$1 \mathrm{~d}$

Figure 16.3 Ontogenetic change and splitting giving rise to the production of new organisms (in the case of organisms), or phylogenetic change and splitting leading to speciation (in the case of species) Based on Hull 1978.

Table 16.3 Applications of Figure 16.3 to Different Cases of Organisms and Species

\begin{tabular}{|c|c|c|c|c|}
\hline \multirow{6}{*}{ Organism } & $\begin{array}{c}\text { Metabolic } \\
\text { change } \\
\text { Ontogenetic } \\
\text { change } \\
\text { Metamorphosis }\end{array}$ & $\begin{array}{c}\text { Equal } \\
\text { splitting (e.g., } \\
\text { transverse } \\
\text { fission in } \\
\text { paramecia) }\end{array}$ & $\begin{array}{c}\text { Budding with } \\
\text { important loss } \\
\text { and } \\
\text { disruption of } \\
\text { internal } \\
\text { organization } \\
\text { (e.g., } \\
\text { strobilization } \\
\text { in Scyphozoa) }\end{array}$ & $\begin{array}{c}\text { Budding with } \\
\text { little loss and } \\
\text { disruption of } \\
\text { internal } \\
\text { organization } \\
\text { (e.g., budding } \\
\text { in Hydrozoa) }\end{array}$ \\
\hline Species & $\begin{array}{c}\text { Change within a } \\
\text { species }\end{array}$ & Splitting & $\begin{array}{c}\text { Speciation, } \\
\text { one species } \\
\text { unchanged, } \\
\text { the other } \\
\text { diverges }\end{array}$ & $\begin{array}{c}\text { A small, } \\
\text { peripheral } \\
\text { isolate brings } \\
\text { about a } \\
\text { genetic } \\
\text { revolution }\end{array}$ \\
\hline
\end{tabular}

definition the observer often faces a continuum of possible situations, but the examples given by Hull help us understand how to apply his view. In case 1a, Hull explains that a living entity can remain the same even if it undergoes a radical change, provided that the continuity between these different states can be established (e.g., a caterpillar becoming a butterfly). Case 1b corresponds to splitting: one individual becomes two individuals, and the initial individual disappears as such. Transverse fission in paramecia is an example. Case 1c corresponds to the appearance of an individual on another individual, with the new individual becoming progressively autonomous. An example is strobilization in certain forms of Scyphozoa (sometimes colloquially called "true jellyfish"). In case 1d, a small part of an individual (contrary to case 1c, this is a part of an individual, not a growing individual on an individual) gains independence and becomes itself a new individual. An example is budding in Hydrozoa (Hydrozoa are Cnidaria that, at least for most of them, have both a polypoid and medusoid stage in their life cycles). Admittedly, it is not trivial to differentiate clearly between $1 \mathrm{c}$ and $1 \mathrm{~d}$, because it is not easy to make the distinction between a growing individual, and a growing part that will become an individual. But the difference between $1 \mathrm{~b}$ on the one hand and $1 \mathrm{c}$ and $1 \mathrm{~d}$ on the other is clear: the case is $1 \mathrm{~b}$ if and only if the initial individual is lost and its internal organization is disrupted. In the two latter cases, the initial individual has certainly lost 
something (a growing new "individual" in 1c, a "small portion" in 1d), but it is still present and its internal organization has remained roughly the same. Naturally, material continuity exists between parents and offspring, but a parent and its offspring are characterized by two different internal organizations, and this is precisely the criterion that makes the difference between the continuity of one being and the continuity of several beings through reproduction.

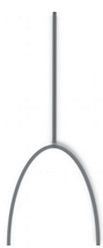

$2 \mathrm{a}$

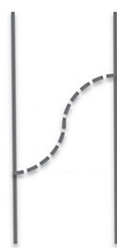

$2 b$

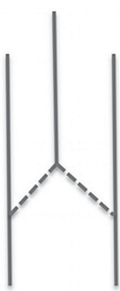

$2 \mathrm{c}$

Figure 16.4 Total or partial merging between organisms, or between species Based on Hull 1978.

A second set of structural changes concerns the merging of two living entities, or of their parts (see figure 16.4 and table 16.4). Here again, it is the examples analyzed by Hull that help clarify his view. In case $2 \mathrm{a}$, two entities fuse to become one single entity, and they remain one entity for a significant time, so the two initial individuals are lost (that is why fusion in amoebas will often not count as an adequate illustration of $2 \mathrm{a}$, while the fusion of two germ cells will do). In case $2 b$, a portion of a first individual becomes a portion of a second individual, and the two individuals continue their existence, but both have changed (the first has lost a part, the second has gained a part). Blood transfusion or bacterial conjugation are good examples. In case 2c, a portion of a first individual and a portion of a second individual merge to form a third (new) individual, while the two initial individuals continue their existence. Sexual reproduction is a good example. If we are interested in species, a good example of $2 \mathrm{~b}$ is introgression, and a good example of $2 \mathrm{c}$ is speciation by polyploidy (a rather common event in plants, for instance).

Table 16.4 Applications of Figure 16.4 to Different Cases of Organisms and Species

\begin{tabular}{|c|c|c|c|}
\hline Organism & $\begin{array}{c}\text { Fusion (e.g. fusion of } \\
\text { sperm and egg) }\end{array}$ & $\begin{array}{c}\text { Endosymbiosis } \\
\text { Blood transfusion } \\
\text { Conjugation }\end{array}$ & Sexual reproduction \\
\hline Species & $\begin{array}{c}\text { Fusion (extremely } \\
\text { unlikely) }\end{array}$ & Introgression & Speciation by polyploidy \\
\hline
\end{tabular}

It should now be apparent that one of the most impressive features of Hull's paper is the richness of its examples. It is indeed these examples that give us the key for understanding Hull's conception of genidentity: because living individuals can change massively and because nothing in them seems to be entirely "fixed" for their entire life, the only way to account for the identity through time of living individuals is to determine to what extent they remain one entity, with an internal organization that remains practically the same or changes progressively. Of course, more precise accounts of what "internal organization" is are needed. But, first, Hull does give us hints as to how this internal organization is maintained or disrupted, in the case of organisms and of species, respectively (for example, the measure of the extension of gene exchange in the case of species: Hull 1978, 349). Second, Hull's main idea seems extremely clear: he 
rejects every conception of identity (of an organism or a species) based on substance (i.e., the idea that "something" remains in an individual despite its changes) and resemblance (i.e., the idea that $\mathrm{X}$ is the same if it looks sufficiently like itself). Importantly, we believe that Hull's view is opposed both to essentialist substantialism (the idea that a permanent "core" or "substrate" of X remains through time) and to the kind of functional substantialism defended, in particular, by Wiggins, because Hull's view is incompatible with the idea that each individual must be understood in relation to a sortal concept, each "sort" being characterized by a common "law of activity" (Wiggins 2011, 57).

Table 16.5 sums up the different conceptions of identity discussed here, their applications to the living world, and some of their proponents.

Table 16.5 Different Concepts of Identity, and Their Application to the Living World

\begin{tabular}{|l|l|l|l|}
\hline Conceptions & Substantialism & Identity-resemblance & Genidentity \\
\hline "Moto" & $\begin{array}{l}\text { Something of X remains } \\
\text { through time }\end{array}$ & X looks sufficiently like itself & $\begin{array}{l}\text { X is defined by } \\
\text { sufficiently } \\
\text { states }\end{array}$ \\
\hline Examples in biology & $\begin{array}{l}\text { - Genetic identity } \\
\text { "Essence" of a species }\end{array}$ & $\begin{array}{l}\text { - Self-resemblance for an } \\
\text { organism } \\
\text { - Species as classes }\end{array}$ & $\begin{array}{l}\text { - Metabolism } \\
\text { - Metamorphosis } \\
\text { - Symbiosis }\end{array}$ \\
\hline Proponents & $\begin{array}{l}\text { Many proponents of } \\
\text { genetic identity (e.g., } \\
\text { Jacob 1982; to some } \\
\text { extent Dawkins 1976) } \\
\text { and genetic essentialism } \\
\text { (see Kripke 1980) }\end{array}$ & $\begin{array}{l}\text { - Hull (1978, 1992) } \\
\text { Boniolo and Carrara } \\
(2004)\end{array}$ \\
\hline
\end{tabular}

So Hull is undeniably the philosopher of biology who offered the strongest defense of the notion of genidentity applied to the living world. Yet we think that it is possible to go even further in Hull's direction.

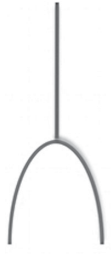

$3 a$

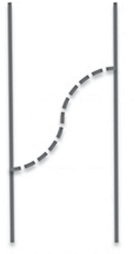

$3 \mathrm{~b}$

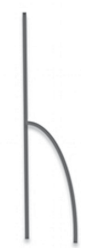

$3 \mathrm{c}$

Figure 16.5 Forms of integration of external biological material, at the organism level or the species level. The first case corresponds to Hull's 2a (fusion), the second to Hull's 2b (integration with continuation), while the third case is an inversion of Hull's 1c. The third case (absent in Hull's analysis) can be called "internalization," and is described in this chapter as an extremely frequent (though long overlooked) phenomenon in nature

Indeed, one additional argument can strengthen Hull's view decisively. It concerns the integration of external components, in particular symbiotic components, into living things. Symbiosis is entirely missing in Hull's picture, except for the rapid mention of the endosymbiotic origin of certain organelles (Hull 1978, 346). This is not really surprising, as it is only recently that symbioses, long thought to be rather 
rare events, have been recognized as extremely widespread in nature (e.g., McFallNgai 2002). Symbiotic elements, and especially symbiotic bacteria, have been shown to decisively influence the ontogeny and phylogeny of many organisms (McFall-Ngai 2002, Pradeu and Carosella 2006, Dupré and O'Malley 2009, Gilbert and Epel 2009, Bouchard 2010, Pradeu 2011). Mammals, for example, are $90 \%$ constituted by bacterial cells and only $10 \%$ by eukaryotic cells, and mutualistic bacteria are indispensable for digestion, immunity, metabolism, and development. The indispensability of symbionts is in fact a phenomenon that can be found in virtually all animals (McFall-Ngai et al. 2013) and plants (Oldroyd 2013). We suggest that the ubiquity of symbiosis strengthens the genidentity view, making Hull's diagrams switch from "weird and rare" to "weird and common," at both the organism and the species levels. Indeed, because symbionts are decisively involved in the ontogeny and phylogeny of most living things, cases $2 \mathrm{a}$ (fusion) or $2 \mathrm{~b}$ (integration with continuation) or, even more frequently, "inverted 1c" (internalization) are extremely widespread in nature (see figure 16.5). In the biological world, the need to integrate foreign (cross-kingdom) living things is the rule, not the exception.

What should one deduce from this importance of symbiosis in nature? In order to understand what a living thing $\mathrm{X}$ is (be it an organism or a species), ${ }^{9}$ one needs to study $\mathrm{X}$ with its symbionts (e.g., Moya et al. 2008), that is, the "heterogeneous organism" or the "heterogeneous species" (Pradeu 2012). In particular, the genome of a living thing X by itself is not sufficient to understand what X is and does. This is what can be called "the revolution of the microbiome" (the "microbiome" refers to the collective genomes of all the microorganisms living in our bodies (Turnbaugh et al. 2007)) or, perhaps more adequately, "the revolution of collective genomes," with metagenomics as a particularly useful set of tools (Dupré and O’Malley 2007, 2009): in most cases, living entities are composite entities, expressing genomes coming from different species, even indeed from different kingdoms (Pradeu 2012, Bapteste 2014). In addition, it is indispensable to study the dynamics of the acquisition of these symbionts, that is, how an organism acquires symbiotic entities through development (McFall-Ngai et al. 2013), and how a species incorporates symbiotic entities through evolution (e.g., Margulis and Sagan 2002, Bright and Bulgheresi 2010). Humans, for example, acquire different types of bacteria throughout their life, and these bacteria influence their development, metabolism, and health (e.g., Scholtens et al. 2012). As a consequence, an organism cannot be biologically defined on the basis of a single and constant genome, or on the basis of self-resemblance, and therefore it seems that only genidentity can account for this dynamic biological identity through time, by capturing the complex processes of symbiont acquisition at the organism level. But the genidentity view is equally pertinent at the species level, as illustrated by many examples, including the growing recognition of different important forms of introgression in prokaryotes (in particular gene transfer agents, conjugative elements, outer membrane vesicles, viruses, plasmids, etc. [Bapteste 2014]), and the study of the role of endogenous viruses in the evolution of their hosts (Roossinck 2011, Stoye 2012). In all these cases, only the idea of a continuous change, in the double sense of a continuity of states and a sufficiently progressive dynamics of change, enables us to follow in details what contributes to the construction of a given living thing. Therefore, the genidentity view seems the best suited to account for the identity through time of living things, and the ubiquity of symbiosis shows that Hull was even more right than could have been appreciated at the time he expressed his view. 
We hope that we have now convinced the reader that the concept of genidentity is potentially extremely relevant to understand identity through time both in physics and in biology. But at this point we would like to draw attention to the way the physical cases studied in section 16.3 shed light on what has been said in the present section about biological cases. From simply potentially relevant, this analogy makes genidentity actually relevant to biology. A preliminary remark is that the physical cases above showed us that the most significant challenge for anyone trying to apply the genidentity view is to determine what process needs to be followed, and how. It seems clear that the same challenge is met in biology. And, here again, it appears that the biologist should follow a causally significant process - for instance (as suggested by Hull) internal organization. At the organism level, internal organization can be measured in terms of intensity of interactions and cohesiveness. A more precise suggestion would be to follow well-specified metabolic interactions, which themselves contribute to the cohesiveness of the organism (see Dupré and O'Malley 2009). Or perhaps one should follow metabolic interactions and higher-level interactions that themselves exert control on these metabolic interactions: for instance, it can be argued that the action of the immune system can play a very important role in the defense of the genidentity view, because the immune system would exert a control on metabolic interactions, and detect and react to any rapid change in the organism (Pradeu 2012, 248-249). At the species level, internal organization can be measured in terms of intensity of gene exchange (again, following Hull), but other forms of transmissions of biological material could be envisioned (membranes, epigenetic marks, etc.) In all these cases, what matters is the causal process that biologists study: how organisms are maintained through time via physiological and metabolic processes, or how species are maintained through time via the interbreeding of organisms.

But the physical cases can shed light on the biological cases examined here in a much more specific way. It seems in fact possible to draw an analogy between the two categories of cases. In drawing this comparison, it is clear that the least useful case taken from physics is the first one, that of the individual soliton (the free individual). The idea of something that would have no interaction with its environment makes no sense in biology, and even the idea of something that would interact with its environment without being itself modified whatsoever by this interaction seems difficult to conceive (though perhaps Dawkins's definition of a selfish gene as an "immortal coil," or "any proportion of chromosomal material that potentially lasts for enough generations to serve as a unit of natural selection," comes close to the idea [Dawkins 1976, 28]). But the second physical case is much more relevant. In the second case, we were interested in a continuously changing wave, and we saw that the key question was, what rate of change makes us consider that $\mathrm{X}$ remains the same individual through time, and what rate of change makes us consider that we do not face the same X? This shift from the question of the degree of change to the question of the rate of change is exactly what illuminates Hull's diagrams. It is, in particular, at the very basis of diagram 1a: X can change, and it can even change massively, as long as the change is progressive enough to make us certain that it is indeed the same individual. But the same principle also lies at the heart 
of all Hull's diagrams: in all cases, it is clear that the rate of "internal organization" disruption will be critical to determine whether we are in the presence of one or two individuals - or perhaps of no individual at all. Finally, the third physical case is also very pertinent to shed light on the biological cases. In one of the cases of the third category, two individuals interact, and it is only the nature of their interaction that enables us to determine whether the two individuals have changed, and whether we still have two individuals anyway-for instance, solitons can fuse, as we saw. It is exactly this idea that lies of the heart of all the cases of biological fusion that we have examined. In order to determine the difference between $2 \mathrm{a}$ (two individuals fuse into one new individual) and $2 \mathrm{~b}$ (one part of an individual $\mathrm{A}$ becomes part of individual $\mathrm{B}$ ), the only resource we have is to determine whether or not the genidentical processes that we were following have been disturbed. In $2 \mathrm{~b}$, we say that the two individuals $\mathrm{A}$ and $\mathrm{B}$ are still the "same" individuals A and B (even though A has lost a part and B has gained this same part) because the rate of change in the internal organization of $A$ has not been significantly disrupted (and the same is true of B). It is also the nature of the interaction that will tell us if we are in a case like 2a (fusion of two individuals; the two "former" individuals no longer exist) or "inverted 1c" (one individual is integrated into an individual; the "host" individual still exists, having been modified but not destroyed by this integration) (see figure 16.5). In the case "inverted 1c," the rate of change has not been massively disturbed by the interaction (though, of course, the change itself can be very significant, as when symbionts can induce a specific morphogenesis in the host), while in case $2 \mathrm{a}$, we talk about a true fusion because a new genidentical process starts, one that will be characterized by its own features, and in particular by its own rate of change, and this new process is not the prolongation of former genidentical processes, as these former processes have been completely disturbed, and hence terminated, because of the interaction (as in two germ cells that fuse and form a zygote).

To conclude this section, we can say that what we have learned by analyzing physical cases of genidentity illuminates in a decisive way the examples taken from biology, because they help us see that, even though biology is often perceived as less "abstract," more "substantial," than physics, it is in fact the same structural characteristics that, in biology and in physics, make it possible to follow entities through time: (1) it is key to determine what exactly is the causal interaction to be followed; (2) what matters is less the degree of change than the rate of change; (3) the most interesting problem concerns the interaction of two genidentically defined individuals, and the way this interaction can disturb their genidentity. Therefore, the concept of genidentity is pertinent not only insofar as it can be applied in interesting ways to both physical and biological cases, but also because it offers a unique opportunity to foster a dialogue between physics and biology.

\subsection{Genidentity and Process Ontology}

An ontology is not just a proposition about the kind of entities that exist in the world, but also a heuristic tool to model the world. As we shall see now, the genidentity view orients us toward an ontology centered on the idea of change and processes, as opposed to an ontology centered on the idea of invariant entities. 
During the scientific revolution, natural philosophers made an important move. As decisively emphasized by Wigner $(1967,3)$, they "devised an artifice which permits the complicated nature of the world to be blamed on something called accidental and thus permits [them] to abstract a domain in which simple laws can be found. The complications are initial conditions; the domain of regularities, laws of nature." It is difficult to overestimate the impact of this heuristic strategy. It allowed physics to become what it is today. One of the reasons it has worked so well is that, in many physical cases, it was possible to fairly well identify the relevant initial conditions, in order to make the discovery of the related law of nature. (For example, think about how Galileo and Newton managed to put aside almost all physical properties to identify the few relevant ones in gravitation phenomena.)

To explain this remarkable success, it is tempting to reify the distinction between laws and initial conditions, in other words between nomic regularities and accidents. For example, we could argue that the laws are independent of the content of the universe, and that, therefore, they are ontologically distinct from accidents that depend on this content. It is what Helen Beebee (2000) called a "governing conception" of laws. If we adopt such a conception, laws are founded on metaphysical invariant features (universals, dispositions...). As brilliantly exposed by Aristotle (Physics 3-5), in an ontological framework focused on what does not change, the main ontological problem becomes the nature of change. Without rejecting the distinction, the metaphysical alternative is to defend a nongoverning conception of laws. In order to make the distinction between laws and accidents not arbitrary, we have to use another kind of invariance to justify the status of laws. For example, in the Ramsay-Mills-Lewis approach, laws are propositions that occupy a special position in the theory structure (axioms, theorems...). What is important to note is that in all these ontological approaches, the focus is on what does not change. Change itself is derivative. Change is understood as what happens when invariance fails. It is not an autonomous metaphysical category.

It is today obvious that the strict distinction between laws and initial conditions is often not very useful in biology. Moreover, in many cases it seems to be an obstacle to the development of biological theories and models. If biology is a historical science, most, if not all, of its propositions are contingent. Should we put all biological knowledge in the column of accidents, therefore in the category of what cannot be adequately explained or modeled? ${ }^{10}$ This seems inappropriate. In the last century, Whitehead (1978) reminded us that one could, and still can, make a metaphysical choice here. Indeed, to follow the Plato-Aristotle trend is not the only possibility. We could put at the center of our ontology the notion of change itself. Consequently, what would be derivative is not change, but the apparent absence of change, in other words regularities and diachronic identity.

This ontological shift of perspective is rarely taken seriously, and this suspicion is understandable given that building an ontology centered on the idea of change and processes is extremely difficult. Past attempts have often been deemed obscure (for example Bergson 1938, Whitehead 1978). Recent moves toward an ontology of processes have been made, especially with regard to the living world (Dupré and O’Malley 2007, 2009, Dupré 2012, Bapteste and Dupré 2013), but a detailed description 
of how such an ontology should be built is still lacking. It is our claim that individuals defined solely by genidentity relation could be a first step toward getting over this problem. Functional genidentity relations, precisely grounded in science (which, as we saw, are not to be understood in mereological terms), could play the central role in a process ontology. In this context, the notion of individual becomes derivative. Needless to say, a lot of work needs to be done before we could claim to have a contender to neoAristotelian approaches, such as Lowe's (2006) or Bird's (2007). As an example, the ontological dependency between processes and events will have to be clarified in a more satisfying way (see Steward 2013, though a process ontology should not be attributed to her). Are processes derivative compared to successions of events, or is the relation between processes and events more equal? Despite the fact that much work is still to be done, we have reasons to be cautiously optimistic. New metaphysical approaches, inspired by current science, for example (Maudlin 2007), are giving us tools to go beyond traditional ontologies. Soon it will not be unreasonable to sustain that processes are ontologically prior, and individuals should be conceived of as specific temporary coalescences of processes. It is scientifically identified processes that will tell us where the individual lies, and what its boundaries are, and not the other way around. We should not start with individuals; we should not build our ontology on the basis of preconceived or phenomenologically determined individuals, because in most cases our intuitive carving of the world into individuals can prove to be misleading in science (in biology, see Hull 1992 and Pradeu 2012; in physics, see Ladyman and Ross 2007 and French and Krause 2006). (Incidentally, we do not claim that descriptive metaphysicsi.e., describing the cognitive structures by which we understand the world-is always wrong. After all, at least pragmatically, our everyday ontology is very efficient. But we definitely sustain that such "anthropocentric" metaphysics tends to exclude innovative and important ontological propositions and revisions, which are often based on our best current science.)

\subsection{Conclusion}

In this chapter, we have defended a genidentity view, that is, the idea that the identity through time of an entity X supervenes on a continuous connection of states. The reason that has led us to this view is partly negative: conceptions of identity based on substance or self-resemblance, which might seem adequate for applications to everyday macroscopic objects, are highly problematic when confronted with cases taken from current physics and biology. In several of the examples studied above, from waves to organisms, nothing really "remains" through time, which makes it impossible to use a substantialist conception of identity; moreover, the overall aspect of the entity under consideration can change so much that it seems impossible to use a conception of identity based on resemblance. For anyone seeking a science-based worldview, therefore, it appears that genidentity is the best-suited view of identity through time. Naturally, the difficulty when applying the genidentity view to specific cases is to determine what exactly needs to be followed and how. Yet we have offered above several examples in which, we believe, it proved possible to address this problem and, therefore, to apply the genidentity view in a fruitful way. We have also shown that 
there are interesting parallels between the applications of genidentity to physical and to biological cases. Finally, we have tried to show that genidentity leads us to suggest an ontology of processes and change rather than an ontology of laws and substances or essences. In this metaphysical conception, processes, we submit, are ontologically prior, and they make possible the delineation of individuals through time, in physics, in biology, and perhaps beyond.

\section{Acknowledgments}

For comments on this chapter, we would like to thank Giovanni Boniolo, Adam Ferner, Steven French, Peter Godfrey-Smith, Matt Haber, Philippe Huneman, and Charles Wolfe.

\section{Notes}

1. Some metaphysicians (e.g., Wiggins 2001) put into question the distinction between synchronic and diachronic identity. Yet this distinction is often useful in a scientific context, both in physics (for example, one could argue that quantum particles are individuals even though they do not perdure) and in biology (for example in the discussion about what it means to be part of a given organism at one moment or at two different moments: e.g., Sober 2000, 154).

2. Maybe not for long, since many physicists and philosophers consider fundamental theories, like quantum field theory, as effective theories, therefore not describing a fundamental ontology, but only applicable in a certain range of parameters. In this context, levels of description and parts-whole relations could come back as important concepts in fundamental physics and not only in applied physics.

3. It is not clear whether, for Lewin, these entities are themselves temporally extended or not. Reichenbach (below) is clearer about this point.

4. Already in 1928, Rudolf Carnap had expressed the same view. He defines genidentity as an "association of various 'thing-states' with one object" (Carnap 1967 [1928], 252).

5. The difficulty of defining wordlines and causation in the quantum context is the main reason that genidentity is not used in current physics. However, these obstacles are not present in classical physics and biology, and this is why we think that Reichenbach's view can be useful in these sciences.

6. More generally, we believe we have good reasons to doubt that the third characteristic needs to be as strong as required by Reichenbach. In Simon Saunders's (2003) terminology, Reichenbach requires that events related by material genidentity refer to an individual that is absolutely discernible. Weakly discernible entities, provided they have a certain dynamics, could also be included in the scope of material genidentity. Since our biological cases are absolutely discernible, we will not push this discussion further.

7. Hull uses "substance" to talk about "material substance" (i.e., remaining materially the same). This is in sharp contrast with what the metaphysical tradition has called a "substance": for Leibniz and Wiggins, for instance, an individual substance can be maintained through time while changing totally its material constituents (Leibniz, New Essays, II, 27). We will show, however, that Hull's critique of substance impacts many forms of substantialism, not just material substantialism.

8. Hull also used the notion of genidentity $(1975,261)$, but it was in a critique of another paper, and Hull was unsympathetic with this notion if used in the context of ordinary language (in contrast with well-formulated scientific theories).

9. In fact, the same is true at other levels of the biological hierarchy, for instance at the level of a cell in a multicellular organism, but for the sake of clarity we stick here to the two main categories explored by Hull. 
10. Even in physics, the scientific status of cosmology (and other "historical" aspects of physics) is also debatable.

\section{Reference}

Aristotle. 1996. Physics. Trans. Robin Waterfield. Oxford: Oxford University Press. Bapteste, Eric. 2014. The Origins of Microbial Adaptations: How Introgressive

Descent, Egalitarian Evolutionary Transitions and Expanded Kin Selection Shape the Network of Life. Frontiers in Microbiology 5. http://journal.frontiersin.org/ Journal/10.3389/fmicb.2014.00083/full.

Bapteste, Eric, and John Dupré. 2013. Towards a Processual Microbial Ontology. Biology and Philosophy 28(2): 379-404.

Beebee, Helen. 2000. The Non-governing Conception of Laws of Nature. Philosophy and Phenomenological Research 61(3): 571-594.

Bergson, Henri. 1938. La Pensée et le Mouvant. Paris: Presses Universitaires de France.

Bird, Alexander. 2007. Nature's Metaphysics: Laws and Properties. Oxford: Oxford University Press.

Boniolo, Giovanni, and Massimiliano Carrara. 2004. On Biological Identity. Biology and Philosophy 19(3): 443-57.

Bouchard, Frédéric. 2010. Symbiosis, Lateral Function Transfer and the (many) Saplings of Life. Biology and Philosophy 25(4): 623-641.

Braillard, Pierre-André, Alexandre Guay, Cyrille Imbert, and Thomas Pradeu. 2011. Une objectivité kaléidoscopique: Construire l'image scientifique du monde. Philosophie 110: 46-71.

Bright, Monika, and Silvia Bulgheresi. 2010. A Complex Journey: Transmission of Microbial Symbionts. Nature Reviews Microbiology 8(3): 218-230.

Carnap, Rudolf. [1928] 1967. The Logical Structure of the World and Pseudoproblems in Philosophy. Berkeley: University of California Press.

Dawkins, Richard. 1976. The Selfish Gene. New York: Oxford University Press. Drazin, Philip G., and Robin Stanley Johnson. 1989. Solitons: An Introduction. Cambridge: Cambridge University Press.

Dupré, John. 2012. Processes of Life: Essays in the Philosophy of Biology. Oxford: Oxford University Press.

Dupré, John, and Maureen O'Malley. 2007. Metagenomics and Biological Ontology. Studies in the History and Philosophy of Science C: Biological and Biomedical Sciences 38: 834-846.

Dupré, John, and Maureen A. O'Malley. 2009. Varieties of Living Things: Life at the Intersection of Lineage and Metabolism. Philosophy and Theory in Biology 1. http://www.philosophyandtheoryinbiology.org/.

French, Steven. 2011. Shifting to Structures in Physics and Biology: A Prophylactic for Promiscuous Realism. Studies in History and Philosophy of Biological and Biomedical Sciences 42(2): 164-173.

French, Steven, and Décio Krause. 2006. Identity in Physics: A Historical, Philosophical and Formal Analysis. Oxford: Oxford University Press.

Gilbert, Scott, and David Epel. 2009. Ecological Developmental Biology: Integrating Epigenetics, Medicine and Evolution. Sunderland: Sinauer Associates.

Hull, David L. 1975. Central Subjects and Historical Narratives. History and Theory 14(3): 253-274.

Hull, David L. 1978. A Matter of Individuality. Philosophy of Science 45(3): 335-360. Hull, David L. 1986. Conceptual Evolution and the Eye of the Octopus. In Logic, Methodology and Philosophy of Science, ed. R. B. Marcus, G. J. W. Dorn, and P. Weingartner, 643-665. Amsterdam: North-Holland.

Hull, David. 1992. Individual. In Keywords in Evolutionary Biology, ed. Evelyn Fox Keller and Elisabeth A. Lloyd, 181-87. Cambridge, MA: Harvard University Press. Jacob, François. 1982. The Possible and the Actual. New York: Pantheon.

Kripke, Saul A. 1980. Naming and Necessity. Cambridge, MA: Harvard University Press.

Leibniz, Gottfried Wilhelm, Freiherr von. [1765] 1916. New Essays Concerning Human Understanding. Chicago: Open Court.

Lewin, Kurt. 1922. Der Begriff der Genese in Physik, Biologie und Entwicklungsge- schichte: Eine Untersuchung zur vergleichenden Wissenschaftslehre. Berlin: Springer-Verlag.

Locke, John. [1694] 1975. An Essay Concerning Human Understanding. 2nd ed. Oxford: Oxford University Press and Clarendon Press.

Lowe, Jonathan E. 2006. The Four-Category Ontology: A Metaphysical Foundation for Natural Science. Oxford: Oxford University Press.

Manton, Nicholas, and Paul Sutcliffe. 2004. Topological Solitons. Cambridge: Cambridge University Press.

Margulis, Lynn, and Dorion Sagan. 2002. Acquiring Genomes: A Theory of the Origins of Species. New York: Basic Books. 
Maudlin, Tim. 2007. The Metaphysics within Physics. New York: Oxford University Press.

McFall-Ngai, Margaret. 2002. Unseen Forces: The Influence of Bacteria on Animal Development. Developmental Biology 242(1): 1-14.

McFall-Ngai, Margaret, et al. 2013. Animals in a Bacterial World: A New Imperative for the Life Sciences. Proceedings of the National Academy of Sciences 110(9): 3229-3236.

Moya, Andrés, et al. 2008. Learning How to Live Together: Genomic Insights into Prokaryote-Animal Symbioses. Nature Reviews Genetics 9: 218-229.

Oldroyd, Giles E. 2013. Speak, Friend, and Enter: Signaling Systems That Promote Beneficial Symbiotic Associations in Plants. Nature Reviews Microbiology 11(4): 252-263.

Padovani, Flavia. 2013. Genidentity and Topology of Time: Kurt Lewin and Hans Reichenbach. In The Berlin Group and the Philosophy of Logical Empiricism, ed. N. Milkov and V. Peckhaus, 97-122. New York: Springer.

Piette, Bernard M. A. G., B. J. Schroers, and W. J. Zakrewski. Dynamics of Baby Skyrmions. Nuclear Physics B 439: 205-235.

Pradeu, Thomas. 2011. A Mixed Self: The Role of Symbiosis in Development. Biological Theory 6(1): 80-88.

Pradeu, Thomas. 2012. The Limits of the Self: Immunology and Biological Identity. New York: Oxford University Press.

Pradeu, Thomas, and Edgardo D. Carosella. 2006. The Self Model and the Conception of Biological Identity in Immunology. Biology and Philosophy 21(2): 235-252.

Quine, Willard Van Orman. 1966. The Ways of Paradox and Other Essays. New York: Random House.

Reichenbach, Hans. [1956] 1971. The Direction of Time. Berkeley: University of California Press.

Reichenbach, Hans. 1958. The Philosophy of Space and Time. New York: Dover Publications.

Roossinck, Marilyn J. 2011. The Good Viruses: Viral Mutualistic Symbioses. Nature Reviews Microbiology 9(2): 99108.

Saunders, Simon. 2003. Physics and Leibniz's Principles. In Symmetries in Physics: Philosophical Reflections, ed. Katherine Brading and Elena Castellani, 289-307. Cambridge: Cambridge University Press.

Scholtens, Petra A., et al. 2012. The Early Settlers: Intestinal Microbiology in Early Life. Annual Review of Food Science and Technology 3: 425-447.

Skyrme, Tony Hilton Royle. 1962. A Unified Field Theory of Mesons and Baryons. Nuclear Physics 31: 556-569.

Smith, Barry, and Kevin Mulligan. 1982. Pieces of a Theory. In Parts and Moments. Studies in Logic and Formal Ontology, ed. Barry Smith, 15-109. Munich: Philosophia Verlag.

Sober, Elliott. 2000. Philosophy of Biology. Boulder, CO: Westview Press.

Soomere, Tarmo. 2011. Solitons Interactions. In Mathematics of Complexity and Dynamical Systems, ed. Robert A. Meyers, 1576-1600. New York: Springer.

Steward, Helen. 2013. Processes, Continuants, and Individuals. Mind 122(487): 781-812.

Stoye, Jonathan P. 2012. Studies of Endogenous Retroviruses Reveal a Continuing Evolutionary Saga. Nature Reviews Microbiology 10(6): 395-406.

Turnbaugh, Peter J., et al. 2007. The Human Microbiome Project: Exploring the

Microbial Part of Ourselves in a Changing World. Nature 449(7164): 804-810. Whitehead, Alfred North. 1978. Process and Reality. New York: Free Press.

Wiggins, David. 1968. On Being in the Same Place at the Same Time. Philosophical Review 77(1): 90-95.

Wiggins, David. 2001. Sameness and Substance Renewed. Cambridge: Cambridge University Press.

Wigner, Eugene P. 1967. Symmetries and Reflections. Woodbridge: Ox Bow Press. 